\title{
Erratum to: Genome-wide association mapping and Identification of candidate genes for fatty acid composition in Brassica napus L. using SNP markers
}

Cunmin $\mathrm{Qu}^{1,2+}$, Ledong Jia ${ }^{1,2+}$, Fuyou Fu ${ }^{3 \dagger}$, Huiyan Zhao ${ }^{1,2}$, Kun Lu ${ }^{1,2}$, Lijuan Wei ${ }^{1,2}$, Xinfu Xu ${ }^{1,2}$, Ying Liang ${ }^{1,2}$, Shimeng $\mathrm{Li}^{1,2}$, Rui Wang ${ }^{1,2^{*}}$ and Jiana $\mathrm{Li}^{1,2^{*}}$

\section{Erratum}

After the publication of this work [1] it was noticed that there was an error with the funding number included in the Funding section where the application number was published instead of the approved funding number. It currently appears as "Chongqing Basic Scientific and advanced technology Research (cstc2015jcyjBX0001, cstc2016shms-ztzx0016 and 21)" and should appear as "Chongqing Basic Scientific and advanced technology Research (cstc2015jcyjBX0001, cstc2016shms-ztzx80020 and ztzx80010)".

\footnotetext{
Author details

${ }^{1}$ Chongqing Engineering Research Center for Rapeseed, College of Agronomy and Biotechnology, Southwest University, Chongqing 400716, China. ${ }^{2}$ Engineering Research Center of South Upland Agriculture of Ministry of Education, Southwest University, Beibei, Chongqing 400716, China. ${ }^{3}$ Department of Botany and Plant Pathology, Purdue University, 915 W. State Street, West Lafayette, IN 47907-2054, USA.
}

Received: 8 May 2017 Accepted: 8 May 2017

Published online: 12 May 2017

\section{Reference}

1. Qu C, Jia L, Fu F, Zhao H, Lu K, Wei L, et al. Genome-wide association mapping and Identification of candidate genes for fatty acid composition in Brassica napus L. using SNP markers. BMC Genomics. 2017;18:232. doi:10. 1186/s12864-017-3607-8.

\footnotetext{
*Correspondence: ruiwang71@163.com; ljn1950@swu.edu.cn

${ }^{\dagger}$ Equal contributors

${ }^{1}$ Chongqing Engineering Research Center for Rapeseed, College of Agronomy and Biotechnology, Southwest University, Chongqing 400716, China
} 